\title{
Analog, Non-Mechanical Beam-Steerer with 80 Degree Field of Regard
}

\author{
Scott R. Davis, "George Farca, Scott D. Rommel, Alan W. Martin and Michael H. Anderson, \\ Vescent Photonics Inc., 4865 E. $41^{\text {st }}$ Ave., Denver CO 80216
}

\begin{abstract}
We are presenting a novel electro-optic architecture for non-mechanical laser beam steering with a demonstrated 80 degrees of steering in a chip-scale package. To our knowledge this is the largest angular coverage ever achieved by non-mechanical means. Even higher angular deflections are possible with our architecture both in the plane of the waveguide and out of the waveguide plane. In the present paper we describe the steering in the plane of the waveguide leaving the out-of-plane scanning mechanism to be detailed in a subsequent publication. In order to realize this performance we exploit an entirely new electro-optic architecture. Specifically, we utilize liquid crystals (LCs), which have the largest known electro-optic response, as an active cladding layer in an LC-waveguide geometry. This architecture exploits the benefits of liquid crystals (large tunable index), while circumventing historic LC limitations. LC-waveguides provide unprecedented macroscopic $(>1 \mathrm{~mm})$ electro-optic phase delays. When combined with patterned electrodes, this provides a truly analog, "Snell's-law-type" beam-steerer. With only two control electrodes we have realized an 80 degree field of view for $1550 \mathrm{~nm}$ light. Furthermore, the waveguide geometry keeps the light from ever coming into contact with an ITO electrode, thereby permitting high optical power transmission. Finally, the beamsteering devices have sub-millisecond response times.
\end{abstract}

Keywords: beamsteering, liquid crystal, spatial light modulators, waveguide, electro-optics, LIDAR.

\section{INTRODUCTION: A NEW ELECTRO OPTIC LASER SCANNER}

Using our innovative electro-optic (EO) waveguide architecture we developed a novel laser scanning device achieving unprecedented angular deflection in a chip-scale package. Wide angular coverage $\left(>+/-40^{\circ}\right)$ is demonstrated with ultra-low power consumption (microwatts), high scanning speed $(<1 \mathrm{msec})$, and analog resolution.

Many new applications require strict limitations in size, weight, and power (SWAP) rendering classic opto-mechanic solutions as inoperative. At less than 15 grams our beam steering solution satisfies even the most stringent requirements with respect to weight, while the ultra-low power consumption ensures hours of autonomous battery operation. Moreover, the immunity to vibrations intrinsic to our EO architecture creates a net advantage over alternative mechanical beamsteering solutions. Pictures of some EO-beamsteering devices are shown in Figure 1 and performance attributes are summarized in Table 1.
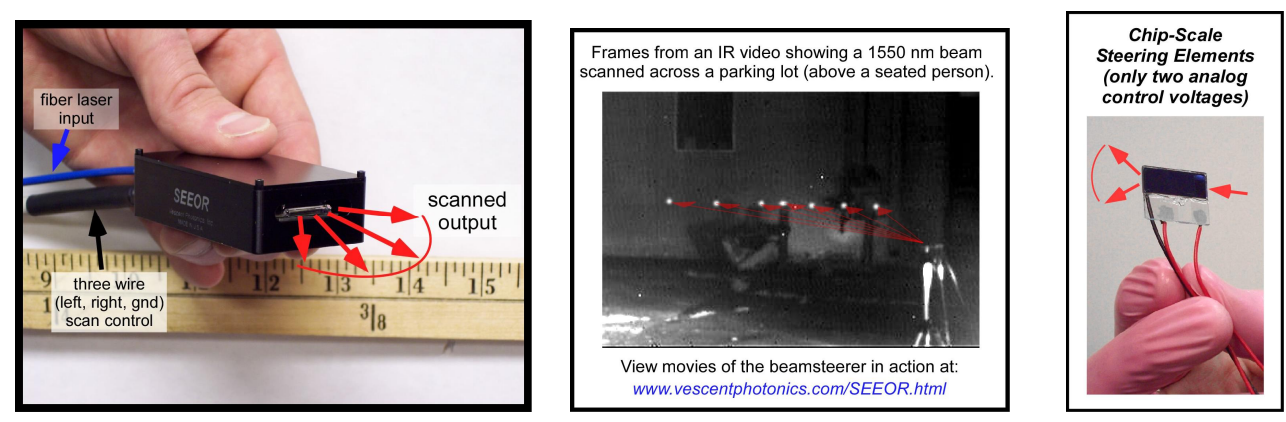

Figure 1: Pictures of Vescent EO beamsteering devices. On left is a packaged device wherein a fiber laser output is controllably voltage steered. In the middle are frames from a movie showing a steered IR spot across a parking lot. On the right is a close up image of the active optical control element, illustrating the simple and compact design.

\footnotetext{
* Corresponding author. Tel.: 303-296-6766; fax: 303-296-6783, E-mail address: davis@vescentphotonics.com
}

Acquisition, Tracking, Pointing, and Laser Systems Technologies XXII, edited by Steven L. Chodos, William E. Thompson, Proc. of SPIE Vol. 6971, 69710G, (2008) · 0277-786X/08/\$18 · doi: 10.1117/12.783766 
Table 1: Performance Attributes of the new Vescent Photonics EO Beamsteerer.

\begin{tabular}{c|c}
\hline \hline Attribute & Performance \\
\hline Size & Compact and Conformal: $<5 \mathrm{~cm}^{3}$ optical head \\
\hline Weight & $<15$ grams for optical head \\
\hline Operating Power & $<100 \mu \mathrm{Watts}$ \\
\hline Angular Coverage & $> \pm 40^{\circ}$ for 1-D sweeps \\
\hline Transmission & $>80 \%$ \\
\hline Control Speed & $<1$ millisecond (full FOV) \\
\hline Steering Resolution & Analog control $(>800$ resolvable spots possible) \\
\hline
\end{tabular}

\section{THE ENABLING INNOVATION}

The challenge of non-mechanical beam control is a long-standing one,${ }^{1-8}$ and has been the subject of extensive past efforts (e.g., The Steered Agile Beam or STAB project ${ }^{9}$ funded by DARPA in 2000). A diverse array of technical approaches have been directed toward this problem including: i) planar electro-optic prisms constructed from KTP, ${ }^{10}$ Lithium Niobate, ${ }^{10}$ ferroelectric domain $\mathrm{LiTaO}_{3},{ }^{7,11}$ and electro-optic polymers, ${ }^{12}$, ii) thermo-optic planar prisms, ${ }^{13}$ iii) diffractive liquid crystal phased arrays ${ }^{5,8}$, and iv) diffractive acousto-optic techniques ${ }^{14}$. Each of these approaches has advantages and drawbacks. Electro-optic crystals are very fast but have extremely small electro-optic coefficients, which means very small steering angles and kilovolts to operate. Furthermore, EO crystals are quite expensive. Liquidcrystal optical-phased array beamsteerers tend to be slow, provide non-continuous diffractive steering, and have a very limited steering range because thick LC layers are problematic. Acousto-optic beamsteerers have a larger steering range but are also diffractive, require very large power and expensive crystals.

Our approach utilizes a new electro-optic architecture that provides unprecedented voltage control over optical phase, which in turn enables a new generation of low-cost EO laser scanners.

\subsection{Giant control over optical phase: LC-waveguides}

Over the past several decades one of the most technically and commercially successful approaches for light control has been liquid-crystal (LC) optics. LCs have the world largest electro-optic response $(\Delta n>0.2$ over 5 volts for a typical LC, which corresponds to $10^{5}-10^{6} \mathrm{pm} / \mathrm{V}$, i.e., several orders of magnitude larger than any other approach), are environmentally stable, and inexpensive. ${ }^{15}$ This has enabled the now $>\$ 50$ billion a year display market. A typical "display-like" LC-optic is shown in Figure 2. The light traverses a thin $(<20 \mu \mathrm{m}) \mathrm{LC}$ layer that is sandwiched between glass sheets. Transparent electrodes are used to apply an electric field, which, in combination with polarizers, may be used to either block or transmit the light.

While undeniably potent for information displays, this traditional LC-optic has two significant limitations. First, the light must transmit through transparent electrodes, which in turn limits the total optical power that may be controlled. Second, and arguably more significant, the LC layer must be extremely thin. The LC- material is rendered a singledomain crystal via thin alignment layers. The LC-molecules
A Conventional Liquid Crystal Optic

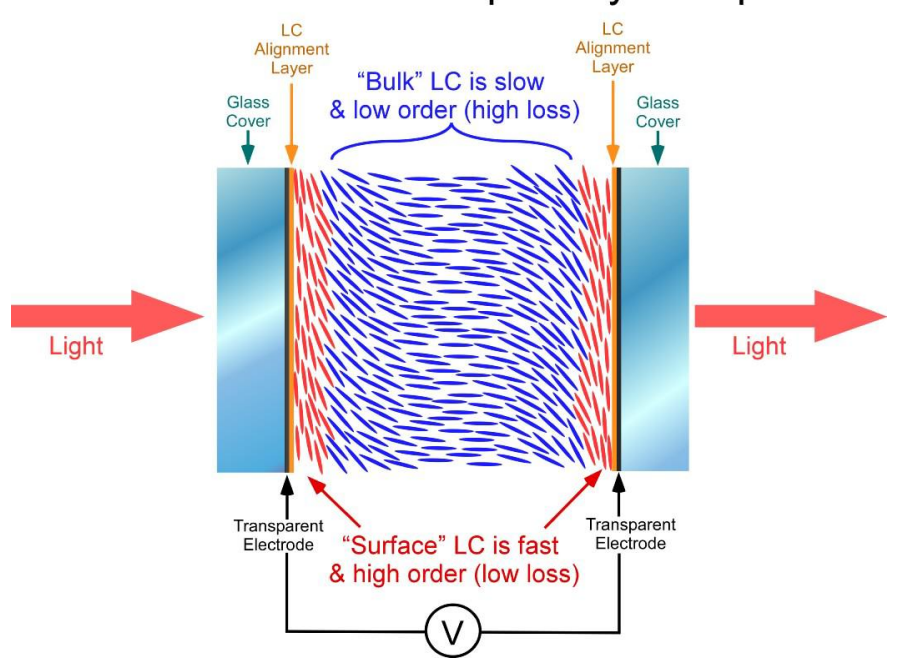

Figure 2: A Typical LC-Optic, such as is used in the ubiquitous LC-Display. 
adjacent these alignment layers (adjacent to the electrodes in Figure 2) are highly ordered, which means low scattering loss, and fast. If one were to make the LC cell thicker, the bulk LC material (middle section in Figure 2) would become prohibitively slow and opaque. Therefore, even though the LC material has a tremendous electro-optic effect, the necessarily short interaction length mitigates this effect. In order to circumvent these limitations we have invented and are developing the LC-clad waveguide architecture, as shown in Figure 3.

\section{Liquid Crystal Clad Waveguides}
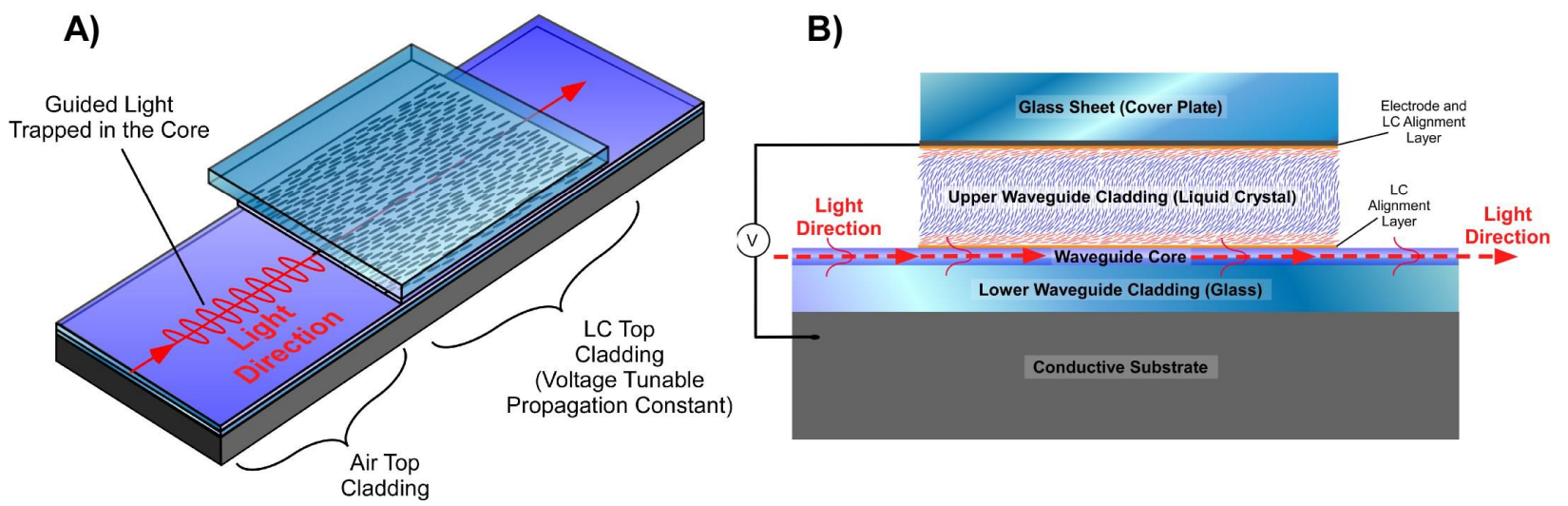

Figure 3: A) The basic geometry of an LC-waveguide. The light is confined to a core and the LC is an electro-optic upper cladding. As the index of refraction of the upper cladding is tuned the "effective index" of the guided mode is also tuned. B) A side view of a liquid crystal waveguide. In a slab waveguide the light is guided in the $x$ dimension, but is free to propagate as Gaussian beams, sheets, or even 1D images in the plane.

Rather than transmit through an LC cell, which by design must be thin (typically $<20 \mu \mathrm{m}$ ), we utilize the LC as an active cladding layer in a waveguide architecture, i.e., the light skims along the surface of an LC layer, as shown in Figure 3. This electro-evanescent architecture circumvents limitations of traditional LC-optics: i) the light never crosses a transparent electrode, ii) the light only interacts with the well-behaved LC-surface layer via the evanescent field, and iii) the interaction length is now decoupled from the LC-layer thickness.

For a given liquid crystal and waveguide structure, we can model the LC upper cladding and the voltage dependent field profile of the guided light. This is shown in the top of Figure 4. Specifically, our model includes: LC surface energy, pre-tilt, elastic coefficients of the LC, electrical properties of the LC (dielectric constants), optical properties of the LC (birefringence), electrode spacing, and electrical properties of the waveguide materials. With this information we can numerically solve for the LC upper cladding index profile as a function of voltage, following an established routine outlined by S. T. Wu. ${ }^{15}$ Then, for a given index profile we can solve Maxwell's equation for the guided mode and determine the effective index. The index modulation is the magnitude of the difference between the effective index at zero volts and the effective index and a higher voltage. This model does an excellent job of predicting the experimental results. Furthermore, the model shows that $\Delta n_{\text {eff }}=0.05$ is possible by using highly-birefringent liquid crystals and by keeping the ratio of core thickness to wavelength less than one. While this represents a four-fold decrease in birefringence from the raw liquid crystal, this is more than offset by a possible 10,000-fold increase in the interaction length. 


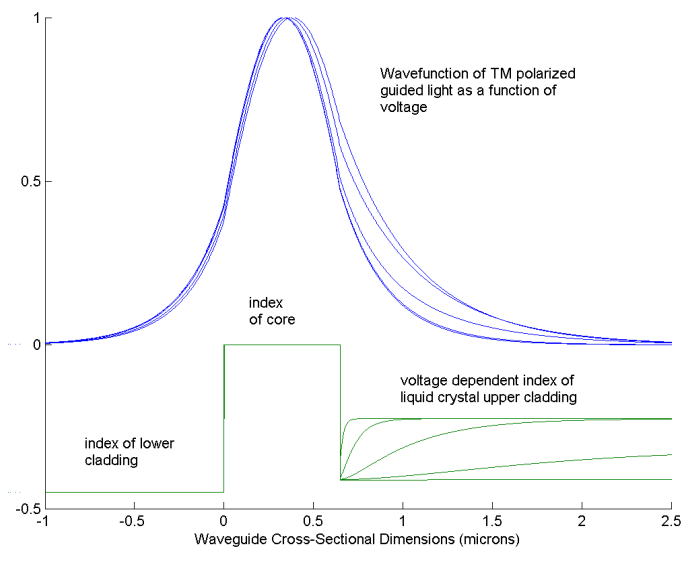

Figure 4: The bottom traces show the calculated index profile of an LC clad waveguide for different applied voltages. As the voltage is increased the index of the upper cladding also increases. The top traces show the intensity profile for TM light as a function of voltage. This was obtained by direct solving of Maxwell's equations for the waveguide boundary conditions.
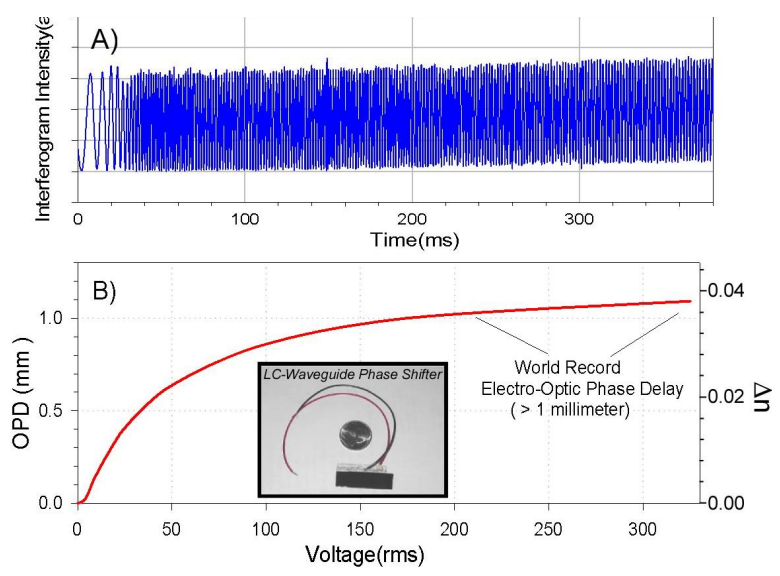

Figure 5: The performance of an LC waveguide filled with a nematic liquid crystal with a birefringence of about $\Delta \mathrm{n} \sim 0.2$. A) The transmission of the LC waveguide between polarizers. The figure was recorded over a longer sweep time so that individual waves could be observed. B) The tunable optical phase delay versus applied voltage. For this device greater than one millimeter of OPD was achieved.

Example operation of a device is given in Figure 5. This device exhibited more than 1 millimeter of voltage tunability over optical phase. We know of no other technology that can provide similar performance. Furthermore, the LC waveguide switching time is faster than normal liquid crystals by about one order of magnitude. Typical relaxation times for LC waveguides are on the order of $500 \mu \mathrm{sec}$.

\subsection{Using LC-waveguides to make new EO laser scanners}

LC-waveguides provide an entirely new approach to electro-optic laser-beamscanning. ${ }^{16}$ The basic concept is shown in Figure 6 wherein a lithographically patterned electrode provides a 1-D prism whose refractive index can be tuned relative to the surrounding area, providing truly analog steering, i.e., it is not diffractive. This lithographically patterned electrode is similar to, albeit much simpler than, the patterned electrodes required for traditional liquid crystal displays. In principle, the pointing precision is only limited by the noise on the control voltage.

To alter the optical functionality one may simply alter the lithographic pattern. The simple example of Figure 6 is for beam deflection, since it contains a non-normal interface. One could also include curved or otherwise shaped interfaces for focusing and/or aberration correction, translation, filtering, or any number of other functions. Figure 7 shows two pertinent examples of lithographically patterned electrodes and how they could be integrated onto a single device. 


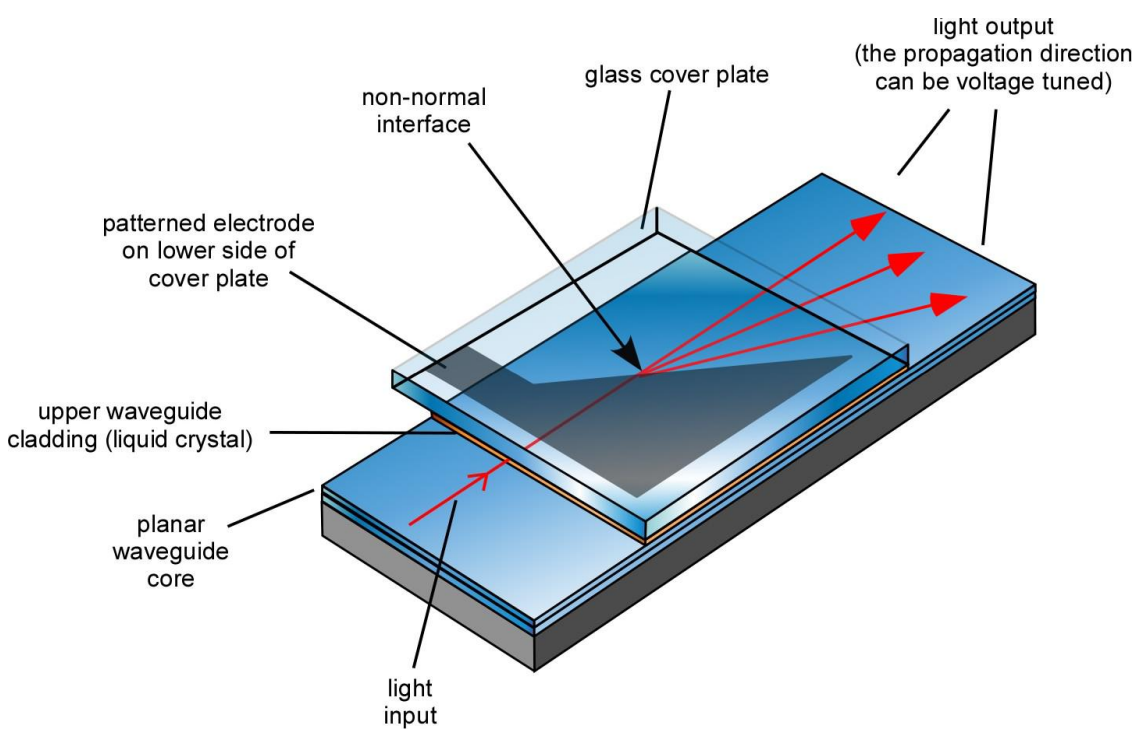

Figure 6: A 1-D LC-waveguide beamsteerer. A single control voltage is applied to a prism-shaped electrode having a non-normal interface to the beam propagation direction. As voltage is applied, the index under the patterned electrode is changed relative to the surrounding area and the beam is steered via Snell's law refraction.

A) Large Angle Steering

\section{Different Electrode Patterns for Different Optical Functionality}

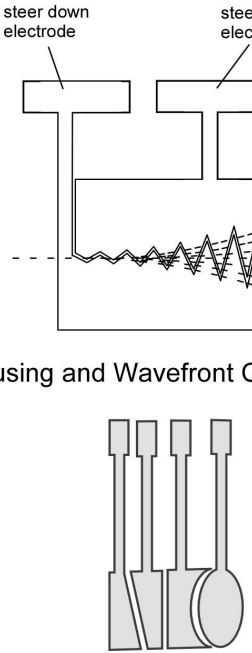

C) Integrated Functionality

B) Focusing and Wavefront Contron
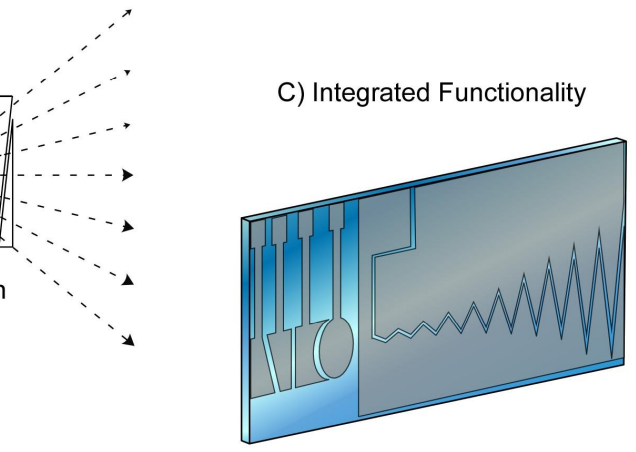

Figure 7: Examples of electrode patterns for different optical functionality. A) A multiple refractive element electrode for increased angular deflection. B) Focusing and conditioning elements for controlling the transmitted wavefront. C) An example of how these might be integrated onto a single adaptive-scanner optical chip. 


\section{DEVICE PERFORMANCE}

\section{1. $\quad \mathbf{8 0}^{\circ}$ of analog electro-optic deflection}

We have designed and built several prototype LC-waveguide beamsteerers. In order to realize a larger deflection angle each steer electrode is patterned to have multiple interfaces in series. In this way the amount of deflection is accumulated along the length of the waveguide. The vertical dimensions of the later electrodes may be expanded to prevent optical clipping of the steered beam. ${ }^{7}$ An example of a multiple interface electrode pattern is shown in Figure 8A. A picture of a prototype waveguide device is shown in Figure 8B, and Figure 8C shows the performance as viewed with a high-gain InGaAs CCD camera. A small amount of non-steered light was deliberately allowed onto the exit card, so an accurate beamsteering angle could be measured. This device exhibited a total analog steer range of over $80^{\circ}$ for a $1 \mathrm{~mm}$ input aperture, with only two control electrodes. To our knowledge this is the largest electro-optic steering ever realized for a two-electrode device. Performance examples of LC-waveguide beamsteerer devices are shown in several short videos online ${ }^{\dagger}$.

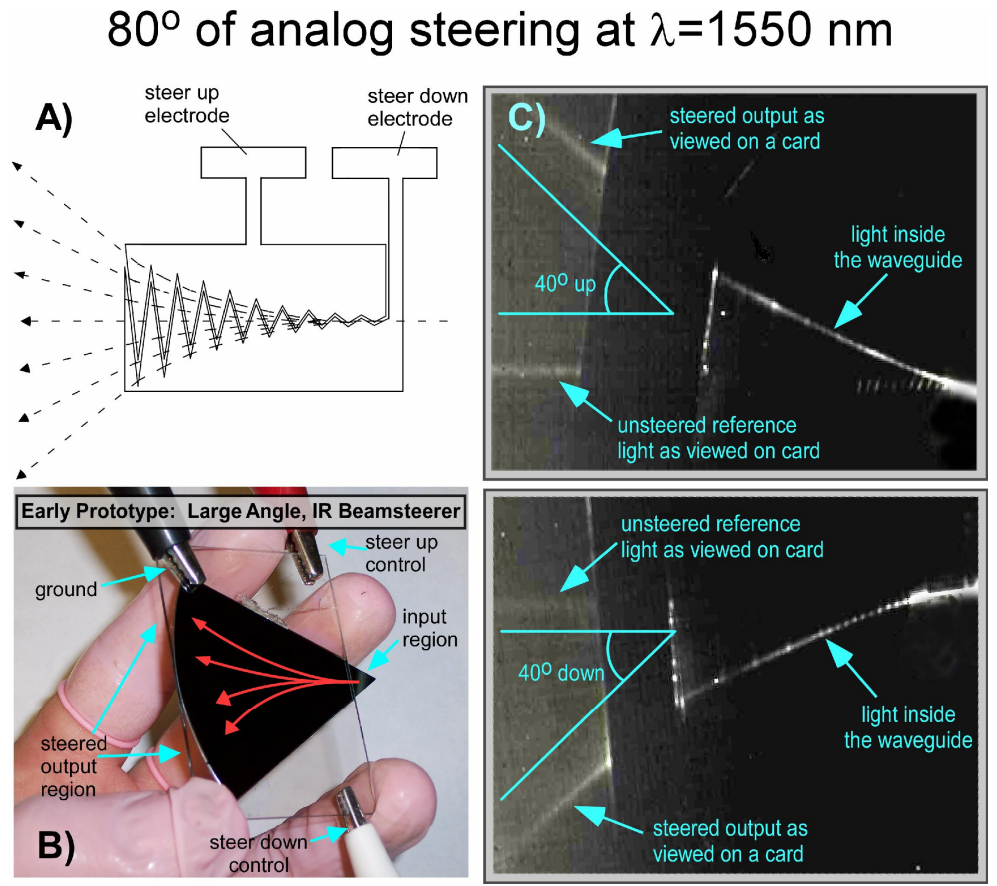

Figure 8: A) The lithographically patterned electrode for controlling beam deflection. B) A picture of a largeangle electro-optic beamsteerer. The red lines are drawn to help guide the eye. This device was made on $\mathrm{Si}$, but it could have been made on glass. C) Images recorded with an InGaAs CCD camera of the prototype beamsteerer in action. The device produced $80^{\circ}$ ( $\pm 40^{\circ}$ of non-mechanical beamsteering, with only two control voltages).

\subsection{Robust photonic packaging for field deployment}

In order for these devices to be useful they must be constructed in a robust package that is suitable for field deployment. We have packaged these devices with a standard FC fiber connector input and a voltage scanned tunable deflection output, as illustrated in Figure 9. The active optical waveguide device is mounted on a ceramic base that serves as both structural support and thermal heat spreading for optional temperature control. Also mounted to the ceramic base are the in-coupling optics and a collimating output lens. The user may control the angle of deflection of the near-diffraction limited output beam via the control electronics. Images of a typical output beam profile are shown in Figure 10.

\footnotetext{
${ }^{\dagger} \mathrm{http}: / /$ www.vescentphotonics.com/SEEOR.html
} 

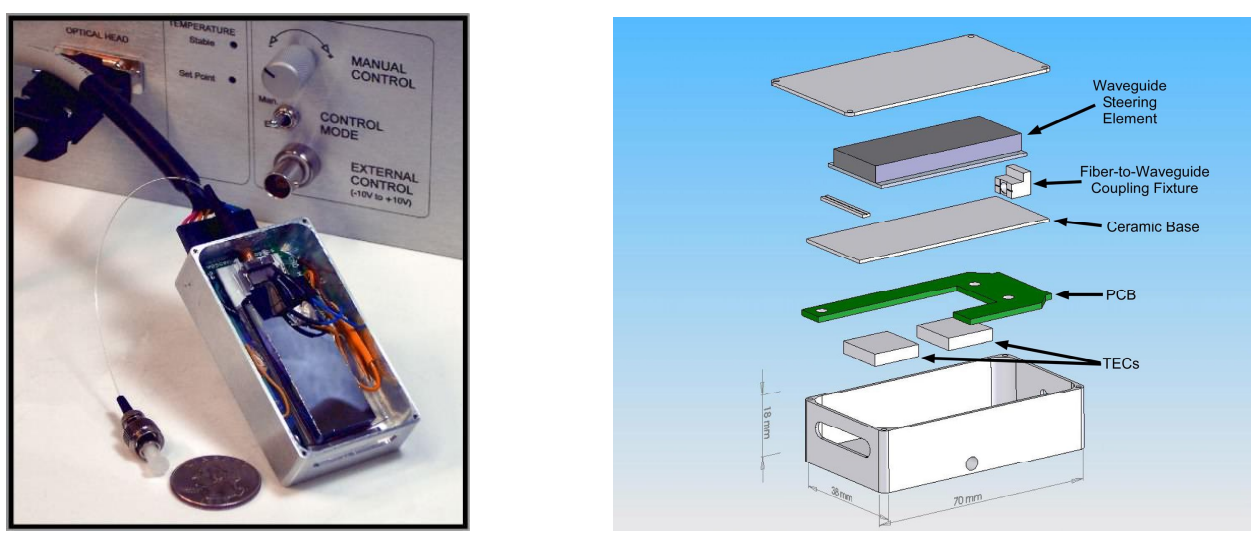

Figure 9: Example of prototype device construction and packaging. The LC-waveguide EO beamsteerer is mounted on a ceramic base, which also holds input coupling optics and an output collimation lens. The user provides a fiberdized light source, which is directly coupled into the device via an FC connector. The output beam is a near-diffraction limited collimated spot the propagation direction of which may be voltage selected by the user.

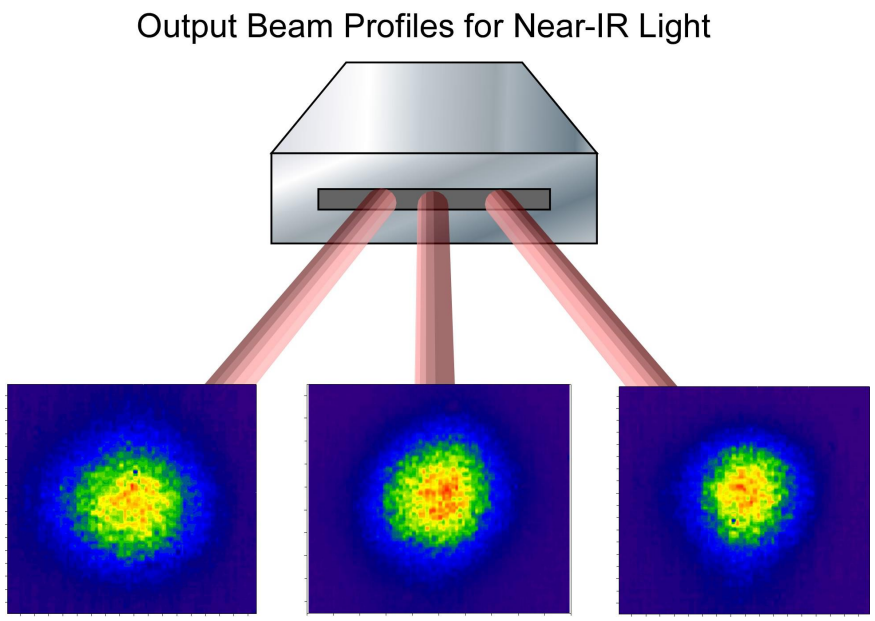

Figure 10: Image scans of a typical near-IR output beam profile from an LC-waveguide EO laser scanner.

The switching speed of liquid crystal devices is generally limited by the "voltage-off" relaxation time. As already mentioned for the LC-waveguide architecture this is greatly reduced due to the close proximity of the liquid crystal molecules to the surface alignment layer. Figure 11 shows two characteristic temporal responses for an LC-waveguide beamsteerer. On the left a photo-diode is placed to detect the maximally steered beam. The voltage is switched on and the beam arrival from no-deflection to maximum-deflection is recorded. As it can be seen the temporal response is approximately $50 \mu \mathrm{sec}$. On the right of Figure 11 the same experiment is repeated except that the beam is now relaxed from a maximum-deflection to a no-deflection point. Even though this operation requires the longest response time of the device, the transition time of $<600 \mu \mathrm{sec}$ still ensures a $>1 \mathrm{kHz}$ full scan rate. This is also the worst-case scenario for random access transition times. If required, optimization of both the LC-waveguide and the electronics can significantly improve upon the speed. 

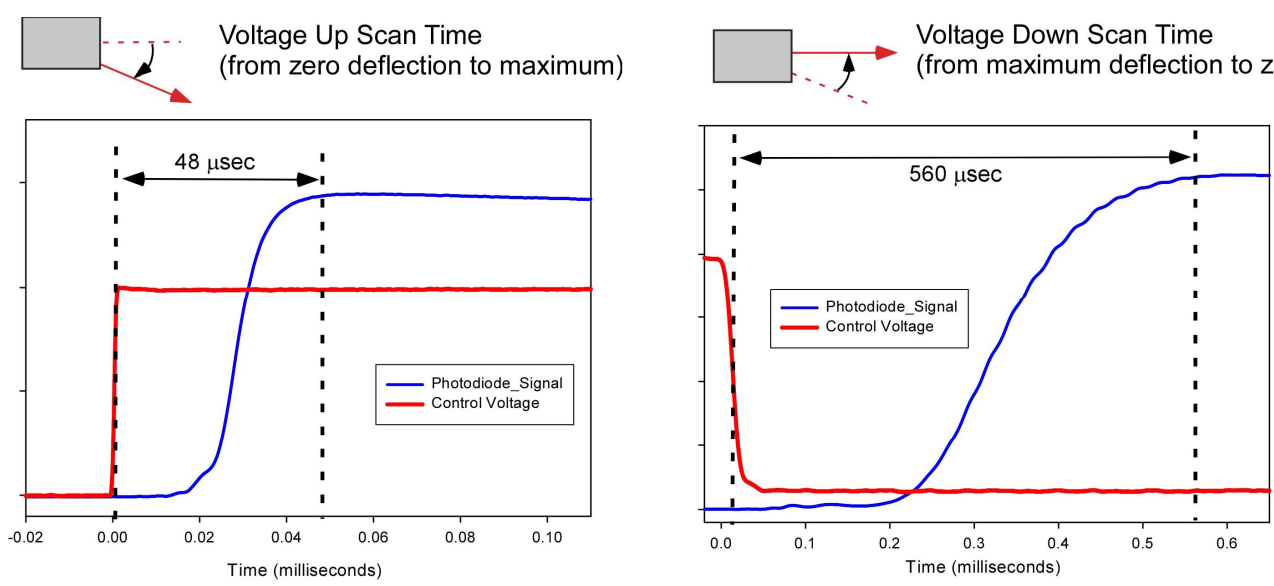

Figure 11: Data scans showing the speed of a prototype LC-waveguide beamsteerer. The figure on the left shows the response time to an up-voltage request. The LC-waveguide can steer from no-deflection to maximum deflection in $\sim 50 \mu \mathrm{sec}$. The figure on the right shows the response time to a voltage-off request. Requiring approximately $600 \mu \mathrm{sec}$, this is the slowest operation mode of the device. All other random access deflection changes will faster.

An infrared video of a beam-steerer in action is available in Video 1. It shows the beam scanning with increased speed, well beyond the frame rate of the camera followed by a sequence of fast random deflections. In the second part it shows the operation outside the laboratory. The IR camera had to be placed behind the device since its field of view is smaller than the angular scanning of the beam-steerer.

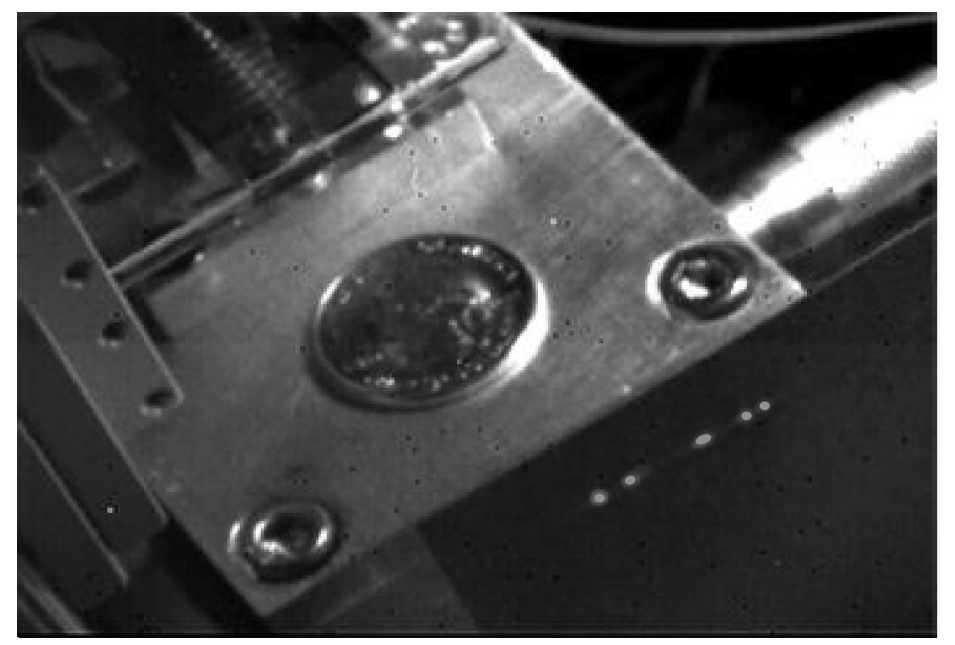

Video 1. Screen caption of the beam-steerer in action. Laboratory operation and deployed performance are shown. http://dx.doi.org/10.1117/12.783766.1

\subsection{Resolution}

There are two kinds of resolution that need be considered. One is the smallest amount one can deflect the beam and the other determines how many spots the scanner can resolve. Because our scanner is analog the first type of resolution is effectively determined by the noise on the drive signal or the bit resolution of a $\mathrm{D} / \mathrm{A}$ converter. An advantage of the analog scanning feature ensures that the entire domain within the field of view can be illuminated such that the point density is determined in principle only by the pulse repetition rate of the laser or the noise on the analog voltage signal.

Dividing the angular stroke by the diffraction angle of a Gaussian beam gives the number of resolvable spots, 


$$
\mathrm{N}_{\text {spots }}=\frac{\theta_{\max }}{\theta_{\text {diff }}} \cong \frac{30^{\circ}}{4 \lambda / \pi d} \approx 800
$$

For LC-waveguide devices under construction we will steer a $3 \mathrm{~mm}$ beam diameter over a $30^{\circ}$ field of regard. For $\lambda=1550 \mathrm{~nm}$ light this translates into approximately 800 resolvable spots.

\section{APPLICATIONS}

Potential applications for active EO scanning systems are increasing in numbers. The dramatic reduction in size, weight, and power combined with the intrinsic ruggedness of the non-mechanical approach enables a wide variety of both military and commercial implementations. Autonomous unmanned (airborne, ground, or sea) vehicle operations are highly dependent on the ability to assess the surrounding topology and the LIDAR/LADAR approach is one of the most promising solutions. Replacing a bulky and power consumptive opto-mechanical scanning assembly will assure extended autonomous capabilities.

As an ultra-compact steerable laser rangefinder it has a wide range of relatively near-term and potentially low-cost military (tactical, theater and strategic) and other (surveillance/homeland security) applications. For example an EOscanning LADAR approach could be readily and cost effectively adapted to fielding of compact eyesafe laser radars, free-space communications capabilities and serve as high-accuracy optical trackers for engaging tactical targets.

Commercial applications include free-space optical communications with pointing adjustment for vibration cancellation, machine and robotic vision, indoor/outdoor mapping, etc. The automobile industry's new concepts of Autonomous (or Adaptive) Cruise Control could also implement this technology once again owing to the low-mass, vibration-insensitive characteristics of a non-mechanical solution.

\section{CONCLUSION AND FUTURE WORK}

In order to exploit the full potential of the EO scanner full 2-D scanning is desirable. The prototypes we are currently building are 1-D scanning systems. As part of future efforts we plan to develop the optimal approach to realize the 2-D angular coverage. One possibility is shown in Figure 12 that utilizes the LC-waveguide architecture to create a tunable out-coupling grating for the second dimension of steering. Once again the high level of refractive index modulation translates into a large angular coverage of the out-coupled beam comparable to the steering demonstrated within the waveguide plane.

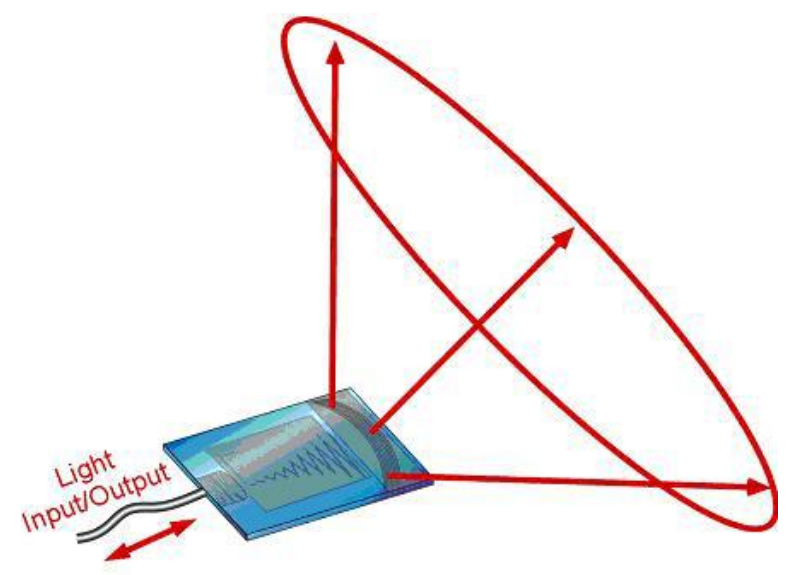

Figure 12: Depiction of full 2-D angular coverage. An LC-tunable out-coupling grating is employed.

Another part of future efforts will be to demonstrate these devices in full LIDAR systems. A critical element of this is collection of the return signal. There are two options for collecting the return beam: back through the waveguide 
scanner or external to the waveguide scanner. Collecting the light back through the same waveguide provides both design simplicity and it enhances tremendously the target discrimination from background radiation. In principle this task will involve similar techniques used in currently commercially available fiber-based LIDAR systems. ${ }^{17}$

The LC-waveguide architecture is a new electro-optic approach that provides unprecedented voltage control over optical phase $(>1 \mathrm{~mm})$. This previously unrealizable level of control makes possible new devices with remarkable performance attributes. To date we have demonstrated: FTIR spectrometers on a chip with $<5 \mathrm{~nm}$ resolution, chip-scale widely tunable lasers (nearly $40 \mathrm{~nm}$ tunability demonstrated), ultra-low power $(<5 \mu \mathrm{W}$ atts) tunable micro-ring filters and Mach-Zehnder switches, and many more. Of particular note to this work we have used LC-waveguides to develop and demonstrate an ultra-wide field of view $\left(80^{\circ}\right)$ non-mechanical laser beamsteerers. All of these devices may be in small LCD-like packages that can ultimately be as low cost as a calculator display.

\section{ACKNOWLEDGMENTS}

This work has been supported by the Air Force Office of Scientific Research under contract number FA9550-06-C-0038 and by the National Science Foundation under award number DMI-0319386. The authors also wish to acknowledge the Colorado Advanced Photonics Technology Center (http://www.captcenter.org), Charles Lee at AFOSR, and Don Snyder at AFRL for their help throughout this development effort. 


\section{REFERENCES}

[1] Chiu, Y., et al., "Shape-Optimized electrooptic beam scanners: Analysis, design, and simulation," J. Lightwave Technol. 17(1), 108-114 (1999).

[2] Finlan, M.J., et al., "Nonmechanical beam steering using spatial multiplexing," Proc. SPIE 3131, 156-164 (1997).

[3] Gahagan, K.T., et al., "Integrated high-power electro-optic lens and large-angle deflector," Appl. Opt. 40(31), 5638-5642 (2001).

[4] Khan, S.A. and N.A. Riza, "Demonstration of 3-dimensional wide angle laser beam scanner using liquid crystals," Opt. Express 12(5), 868-882 (2004).

[5] McManamon, P., "An overview of optical phased array technology and status," Proc, SPIE 5947, 152-161 (2005).

[6] Revelli, J.F., "High-resolution electrooptic surface prism waveguide deflector: an analysis," Appl. Opt. 19(3), 389-397 (1980).

[7] Scrymgeour, D.A., et al., "Large-angle electro-optic laser scanner on LiTaO3 fabricated by in situ monitoring of ferroelectric-domain micropatterning," Appl. Opt. 40(34), 6236-6241 (2001).

[8] Stockley, J.E., et al., "Broadband beam steering," Proc. SPIE 3131, 111-123 (1997).

[9] http://www.darpa.mil/mto/stab/.

[10] Chiu, Y., et al., "Design and Simulation of Waveguide Electrooptic Beam Deflectors," J. Lightwave Technol. 13(10), 2049-2052 (1995).

[11] Scrymgeour, D.A., et al., "Phased-array electro-optic steering of large aperture laser beams using ferroelectrics," Appl. Phys. Lett. 86(21), 211113-211119 (2005).

[12] Sun, L., et al., "Polymeric waveguide prism-based electro-optic beam deflector." Opt. Eng. 40(7), $1217-1222(2001)$

[13] Kim, J.-h., et al., "Polymer-based thermo-optic waveguide beam deflector with novel dual foldedthin-strip heating electrodes," Opt. Eng. 42(3), 620-624 (2003).

[14] Yariv, A., [Quantum Electronics, $3^{\text {rd }}$ ed], John Wiley \& Sons, New York, (1989).

[15] Khoo, I.-C. and Wu, S.-T., [Optics and nonlinear optics of liquid crystals], World Scientific Publishing, Singapore, (1993).

[16] Anderson, M., S. Davis, and S. Rommel, "Liquid Crystal Waveguide having Refractive Shapes for Dynamically Controlling Light" USPTO Patent Application, Vescent Photonics, Inc., US (2004).

[17] Wher, A. and U. Lohr, "Airborne laser scanning -- an introduction and overview." ISPRS J. of Photogrammetry \& Remote Sensing 54, 68-82 (1999). 